\title{
The Hidden Consequences of Ventilator Management Decisions
}

\author{
Rolf D Hubmayr MD and Richard A Oeckler MD PhD
}

\begin{abstract}
In the following perspective, we will highlight seemingly remote, downstream consequences of common ventilator management decisions. For example, a change in PEEP may alter venous return, blood pressure, cardiac output, arterial and venous blood gas tensions, metabolic rate, respiratory sensations, breathing pattern, and the work of breathing. If providers consider any of these changes dangerous or maladaptive, they may initiate additional interventions in the form of vasoactive agents, intravenous fluids, and/or sedatives, all of which have their own risk/benefit profile. The approach to such co-interventions is rarely addressed even in well-designed large clinical trials. Therefore, it is often impossible to infer intervention-specific mechanisms of action and/or identify the phenotype of responders and nonresponders in such trials. On the flip side, in preclinical research intended to uncover mechanisms, experimental animals are rarely treated the way a critically ill patient would be. For respiratory therapists, this knowledge gap stresses the imperative to think beyond the lungs and to communicate ventilator management decisions with all members of the healthcare team. Key words: mechanical ventilation; adverse effects; lung injury; supportive care. [Respir Care 2014;59(8):1302-1305. (C) 2014 Daedalus Enterprises]
\end{abstract}

\section{Introduction}

A casual search of the biomedical literature of the last 5 years generates $>100,000$ hits for articles pertaining to "mechanical ventilation." This number is not surprising, given the need for respiratory support during surgery and critical illness, the advances in sensor and microprocessor technology, and the growing appreciation of mechanobiology in the pathogenesis of lung injury and repair. The concern for ventilator-induced lung injury (VILI) has mo-

The authors are affiliated with the Department of Pulmonary and Critical Care Medicine, Mayo Clinic, Rochester, Minnesota.

Dr Hubmayr presented a version of this paper as the 40th Donald F Egan Scientific Memorial Lecture at the 59th AARC Congress, held November 16-19, 2013, in Anaheim, California.

Dr Hubmayr was supported in part by grant R01 HL63178 from the National Institutes of Health and a grant from the Annenberg Foundation. $\mathrm{He}$ serves as a consultant for Philips Medical and chairs the advisory board of Respithera. Dr Oeckler was supported by a grant from the Parker B Francis Foundation.

Correspondence: Rolf D Hubmayr MD, 200 First Street SW, Rochester, MN 55905. E-mail: rhubmayr@mayo.edu.

DOI: $10.4187 /$ respcare.03366 tivated numerous preclinical and clinical trials focused on optimal ventilator settings and established low tidal volume $\left(\mathrm{V}_{\mathrm{T}}\right)$ ventilation as the standard of care for patients with ARDS. ${ }^{1}$ Although there is a general consensus that large parenchymal deformations induce a pro-inflammatory immune response, the specific means to minimize biophysical lung damage remains a topic of active investigation and debate. Preclinical studies tend to focus on the topographical distributions of parenchymal stress and strain and on the effects of breathing mode, ventilator settings, and body posture upon them. Clinical trials, in turn, examine the effects of the very same variables on patientcentric outcomes. As in any integrated system, an intervention directed at one system component can trigger remote or global system responses. For ventilator management, such responses can manifest as hemodynamic instability, shortness of breath, pain, and agitation.

\section{$V_{T}$ and Lung Injury}

The $\mathrm{V}_{\mathrm{T}}$ setting is not only a critical determinant of arterial oxygenation and alveolar ventilation, but is also the most important risk factor of VILI. ${ }^{2}$ Therefore, many providers limit $\mathrm{V}_{\mathrm{T}}$ to $<8 \mathrm{~mL} / \mathrm{kg}$ predicted body weight even in patients with normal lungs. ${ }^{3}$ This differs greatly from historic norms, in which $\mathrm{V}_{\mathrm{T}}$ as large as $15 \mathrm{~mL} / \mathrm{kg}$ 
actual body weight were the standard of care. The reasons why early practitioners of mechanical ventilation chose to set $\mathrm{V}_{\mathrm{T}}$ to supraphysiologic levels are informative. For one, the discipline of critical care arose from the need to provide respiratory support following surgery. Anesthetists had learned that large tidal inflations were needed to recruit atelectatic lung during inhalational anesthesia and thereby prevent hypoxemia. ${ }^{4}$ Although it is now understood that PEEP is the preferred means of preventing perioperative gas absorption atelectasis, ${ }^{5}$ that insight was not appreciated at the time. Moreover, the use of PEEP in this setting can frequently cause hypotension due to the effect of anesthesia upon venous capacitance and vascular tone. ${ }^{6}$ Therefore, some providers choose to limit the use of PEEP despite strong clinical evidence that lung-protective ventilator settings during surgery are efficacious and associated with fewer postoperative complications. ${ }^{7}$ Choosing to protect the lungs by applying high PEEP while accepting the cardiovascular risks associated with the aggressive use of inotropes and vasoactive agents is particularly challenging in morbidly obese patients. ${ }^{8}$

A second reason for the acceptance of large $\mathrm{V}_{\mathrm{T}}$ settings as the norm during the early days of mechanical ventilation was air hunger, which even normocapnic patients may perceive while supported in volume modes. The loss of variability in breath-to-breath amplitude and timing can generate an error signal, which manifests as dyspnea. Dyspnea, in turn, may be relieved by large tidal inflations of the chest on account of vagal afferent stimulation ${ }^{9}$ and is another reason why the use of sedatives and narcotics is commonplace in the care of mechanically ventilated patients.

Because $\mathrm{V}_{\mathrm{T}}$, and thus lung strain-a dimensionless measure of parenchymal stretch/deformation - are central to the pathogenesis of VILI, it only makes sense to employ volume modes in patients with the highest risk of injury. However, the literature contains numerous, largely unsubstantiated claims that certain pressure modes are superior to volume controlled modes for patients with ARDS. ${ }^{10}$ In general, benefit has been inferred from improvements in arterial oxygenation and better patient/ventilator synchrony. For the most part, these improvements can be traced to an increase in $\mathrm{V}_{\mathrm{T}}$ and may therefore not be inherent to ventilation mode per se. Unless patients are heavily sedated and paralyzed, pressure modes do preserve some of the biologic variability in breathing pattern. However, mode-related differences in ventilation and oxygenation need not convey long-term benefit if they are associated with an increased VILI risk.

\section{$\mathbf{V}_{\mathrm{T}}$ Limitation During Spontaneous Breathing}

There is a great deal of uncertainty as to whether the goals of lung protection, namely the restriction of $\mathrm{V}_{\mathrm{T}}$ to $<8 \mathrm{~mL} / \mathrm{kg}$ predicted body weight and the application of

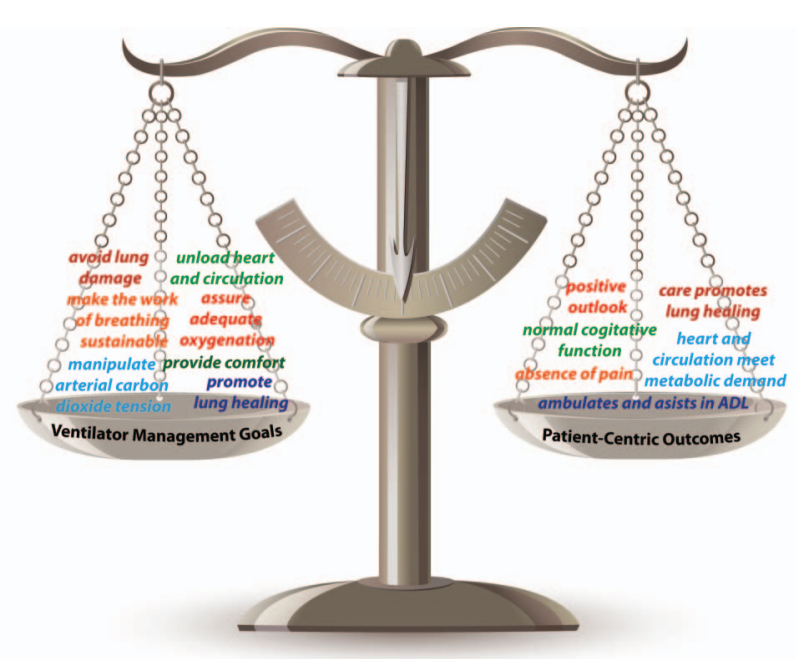

Fig. 1. Balancing ventilator management goals and patient-centric outcomes.

higher PEEP settings, should be applied to spontaneously breathing ARDS patients as well. It is not uncommon for patients to exceed safe $\mathrm{V}_{\mathrm{T}}$ limits early on, while supported with noninvasive ventilation, or later in the course of their disease during spontaneous breathing trials. In both instances, the decision to impose safe $\mathrm{V}_{\mathrm{T}}$ and optimal PEEP settings assumes that the risk of causing lung injury outweighs the risks associated with prolonged intubation and the use of sedatives, narcotics, and paralytics (Fig. 1).

Although there are no specific quantitative data to allow providers to precisely balance these risks, current knowledge of biophysical lung injury mechanisms does suggest that the risk of VILI is not specific to breathing mode and applies equally to mechanical ventilation and spontaneous breathing. A recent report suggests that diaphragm-apposed lung regions could be exposed to injurious deformations during strong inspiratory ventilator triggering efforts. ${ }^{11,12}$ In fact, there is growing evidence that the use of paralytics during the initial stages of ARDS management is associated with improved patient survival. ${ }^{13}$ Therefore, it seems unwise to delay intubation in a spontaneously breathing or noninvasively ventilated patient, who maintains acceptable arterial oxygen tensions only at the expense of distress and persistent hyperventilation. Neither the mechanisms of action nor the optimal duration of neuromuscular blockade in patients with severe ARDS are firmly established. The clinical trial, which suggested efficacy, arbitrarily set the duration of neuromuscular blockade to $48 \mathrm{~h}$. Better data on the optimum timeframe of this intervention would be helpful, because prolonged use of paralytics is associated with muscle wasting, tends to be applied in conjunction with deep sedation, and therefore delays any chance for early physical rehabilitation and risks late neuropsychiatric complications. ${ }^{14-16}$ 


\section{Lung Protection During Ventilator Liberation}

The optimal approach to lung protection during the weaning phase is even less clear. In particular, patients who had been deeply sedated and are prone to agitated delirium tend to take very large tidal breaths while they are assessed for liberation from mechanical ventilation. Nonetheless, their VILI risk is presumably lower than it had been at the outset, because the alveolar edema that amplifies $\mathrm{V}_{\mathrm{T}^{-}}$-dependent interfacial injury mechanisms has by then undergone a phase transition from a liquid to a gel. ${ }^{17,18}$ In other words, the conditions that had favored injury by so-called atelectrauma may no longer prevail. ${ }^{19}$ Despite the low rapid-shallow breathing index, some of these patients may not meet criteria for extubation due to persistent encephalopathy or high PEEP requirements. Many practitioners ignore less-than-ideal $V_{T}$ under these circumstances, because they consider the risks associated with keeping a patient in a drug-induced encephalopathic state to be unacceptable.

\section{Breath Stacking}

One of the more common concerns in patients who are ventilated with lung-protective $\mathrm{V}_{\mathrm{T}}$ in a volume mode is breath stacking. This happens whenever the neural inspiratory time, that is, the time during which inspiratory motor units are active, exceeds the machine-set inspiratory time or when spontaneous inspiratory activity is coupled in a peculiar entrainment pattern to machine inflations. ${ }^{20,21}$ Although switching from a volume mode to a pressure mode is likely to eliminate most forms of breath stacking, it typically does so by allowing $\mathrm{V}_{\mathrm{T}}$ to rise to potentially injurious levels. Under such circumstances, the provider is faced with several choices: (1) to accept the $\mathrm{V}_{\mathrm{T}}$-associated VILI risk; (2) to enforce lung protection through the use of sedatives, narcotics, and/or neuromuscular blocking agents; or (3) to enforce lung protection by prolonging the machine-set inspiratory time, that is, by reducing inspiratory flow.

In healthy subjects with intact vagal afferent reflexes, reducing inspiratory flow would simply prolong neural inspiratory time further and would likely be counterproductive. ${ }^{22}$ However, patients who are stressed often lose neuromechanical modulation of respiratory motor output. Nevertheless, reducing flow in hypercapnic patients rarely eliminates breath stacking. When it does eliminate it, it does so at the expense of increased and potentially exhausting respiratory muscle efforts. Such efforts can be associated with substantial lung and chest wall distortions and may cause injury to diaphragm-apposed lung regions, even at recommended $\mathrm{V}_{\mathrm{T}}{ }^{11}$

\section{Prone Position}

Recent meta-analyses and a prospective clinical trial have rekindled interest in the prone position as a lung- protective intervention. ${ }^{23,24}$ Based upon available evidence, the decision to place a patient in the prone position should be reserved for individuals with the most severely injured lungs and is therefore often employed from the outset, in conjunction with deep sedation and induced muscle paralysis. However, if prone positioning is to improve outcome, it requires a long-term commitment to this strategy, that is, the maintenance of the prone posture for many days with only brief daily supine holidays. ${ }^{24}$ Because so little is known about the long-term benefits of early mobilization ${ }^{25}$ or about managing ventilated patients without the adjunct use of sedatives, ${ }^{26}$ the unintended consequences of prone lung protection could prove costly. As is true for virtually all clinical intervention trials to date in which specific ventilator management approaches were found efficacious, the comparison groups tended to involve well-sedated patients, who rarely left their bed.

\section{Recruitment Strategies}

The sustained recruitment of airless lung units is one of the central tenets of lung-protective mechanical ventilation. Recruitment strategies seek to reduce parenchymal strain by distributing inspired gas across a greater number of lung units ${ }^{27}$ and serve to protect the lungs from socalled atelectrauma by preventing liquid bridge formation in small airways and airspaces. ${ }^{19,28}$ Recruitment generally requires a transient inflation of the lungs to high pressures and volumes, followed by the application of PEEP to maintain the recruitment gains. An in-depth discussion of the merits of different recruitment strategies is beyond the scope of this perspective. Nevertheless, practitioners need to understand that not all changes in respiratory mechanics that accompany recruitment maneuvers mean that previously closed lung units have in fact opened, ${ }^{29}$ or that recruitment maneuver-associated changes in $\mathrm{P}_{\mathrm{aO}_{2}}$ are necessarily biomarkers of long term benefit.

Those who define the optimal PEEP as the end-expiratory pressure at which respiratory system compliance is maintained at the highest possible value ${ }^{30}$ must be comfortable having to support blood pressure and cardiac output by pharmacologic means. Not only will high levels of PEEP increase pleural pressure and thereby lower venous return, PEEP can also precipitate right heart failure on account of its effect on pulmonary vascular resistance and right ventricular afterload. These conditions must be recognized and differentiated because they require distinct interventions, and can prove challenging even in experienced hands.

A recently published report, which suggested that the early application of high frequency oscillatory ventilation (HFOV) in patients with ARDS may cause harm, highlights this challenge. ${ }^{31}$ In-hospital mortality was $47 \%$ in the HFOV group, as compared with $35 \%$ in the control 


\section{Consequences of Ventilator Management Decisions}

group (relative risk of death with HFOV, 1.33; 95\% CI, $1.09-1.64, P=.005$ ). Because HFOV represents arguably the most aggressive lung recruitment strategy, more subjects randomized to the HFOV arm required vasoactive drugs and did so for substantially longer periods of time. It would be inappropriate to attribute the mortality difference between HFOV and conventional care entirely to adverse cardiovascular effects associated with an overaggressive recruitment strategy. At the same time, this hypothesis cannot be rejected offhand.

\section{Conclusions}

To conclude, the examples in this perspective seek to emphasize that ventilator management decisions must be made in anticipation of required co-interventions and with consideration of their associated risks and benefits. Affected domains are both physical and psychological. The examples point out significant knowledge gaps surrounding current standards of supportive care and should serve as a reminder that benefit of ventilator management decisions should not be judged by their effects on lung function alone.

\section{REFERENCES}

1. Ventilation with lower tidal volumes as compared with traditional tidal volumes for acute lung injury and the acute respiratory distress syndrome. The Acute Respiratory Distress Syndrome Network. N Engl J Med 2000;342(18):1301-1308.

2. Slutsky AS, Ranieri VM. Ventilator-induced lung injury. N Engl J Med 2013;369(22):2126-2136.

3. Hubmayr RD. Point: is low tidal volume mechanical ventilation preferred for all patients on ventilation? Yes. Chest 2011;140(1):9-11.

4. Bendixen HH, Hedley-Whyte J, Laver MB. Impaired oxygenation in surgical patients during general anesthesia with controlled ventilation: a concept of atelectasis. N Engl J Med 1963;269:991-996.

5. Rothen HU, Sporre B, Engberg G, Wegenius G, Reber A, Hedenstierna G. Prevention of atelectasis during general anaesthesia. Lancet 1995;345(8962):1387-1391.

6. Longnecker DE. Effects of general anesthetics on the microcirculation. Microcirc Endothelium Lymphatics 1984;1(2):129-150.

7. Futier E, Constantin JM, Paugam-Burtz C, Pascal J, Eurin M, Neuschwander A, et al. A trial of intraoperative low-tidal-volume ventilation in abdominal surgery. N Engl J Med 2013;369(5):428437.

8. Sprung J, Whalley DG, Falcone T, Wilks W, Navratil JE, Bourke DL. The effects of tidal volume and respiratory rate on oxygenation and respiratory mechanics during laparoscopy in morbidly obese patients. Anesth Analg 2003;97(1):268-274;table of contents.

9. Manning HL, Shea SA, Schwartzstein RM, Lansing RW, Brown R, Banzett RB. Reduced tidal volume increases "air hunger" at fixed $\mathrm{PCO}_{2}$ in ventilated quadriplegics. Respir Physiol 1992;90(1):19-30.

10. Kallet RH. Patient-ventilator interaction during acute lung injury, and the role of spontaneous breathing; part 2: airway pressure release ventilation. Respir Care 56(2):190-203, 2011; discussion 203-196.

11. Yoshida T, Torsani V, Gomes S, De Santis RR, Beraldo MA, Costa EL, et al. Spontaneous effort causes occult pendelluft during mechanical ventilation. Am J Respir Crit Care Med 2013;188(12):1420-1427.
12. Hubmayr RD. Volutrauma and regional ventilation revisited. Am J Respir Crit Care Med 2013;188(12):1388-1389.

13. Papazian L, Forel JM, Gacouin A, Penot-Ragon C, Perrin G, Loundou A, et al. Neuromuscular blockers in early acute respiratory distress syndrome. N Engl J Med 2010;363(12):1107-1116.

14. Fan E, Dowdy DW, Colantuoni E, Mendez-Tellez PA, Sevransky JE, Shanholtz C, et al. Physical complications in acute lung injury survivors: a 2-year longitudinal prospective study. Crit Care Med 2014; 42(4):849-859.

15. Alhazzani W, Alshahrani M, Jaeschke R, Forel JM, Papazian L, Sevransky J, et al. Neuromuscular blocking agents in acute respiratory distress syndrome: a systematic review and meta-analysis of randomized controlled trials. Crit Care 2013;17(2):R43.

16. Brummel NE, Ely EW. Sedation level and the prevalence of delirium. Intensive Care Med 2014;40(1):135.

17. Hussein O, Walters B, Stroetz R, Valencia P, McCall D, Hubmayr RD. Biophysical determinants of alveolar epithelial plasma membrane wounding associated with mechanical ventilation. Am J Physiol Lung Cell Mol Physiol 2013;305(7):L478-L484 .

18. McGee KP, Mariappan YK, Hubmayr RD, Carter RE, Bao Z, Levin DL, et al. Magnetic resonance assessment of parenchymal elasticity in normal and edematous, ventilator-injured lung. J Appl Physiol 2012;113(4):666-676.

19. Muscedere JG, Mullen JB, Gan K, Slutsky AS. Tidal ventilation at low airway pressures can augment lung injury. Am J Respir Crit Care Med 1994;149(5):1327-1334.

20. Akoumianaki E, Lyazidi A, Rey N, Matamis D, Perez-Martinez N, Giraud R, et al. Mechanical ventilation-induced reverse-triggered breaths: a frequently unrecognized form of neuromechanical coupling. Chest 2013;143(4):927-938

21. Simon PM, Zurob AS, Wies WM, Leiter JC, Hubmayr RD. Entrainment of respiration in humans by periodic lung inflations. Effect of state and $\mathrm{CO}_{2}$. Am J Respir Crit Care Med 1999;160(3):950-960.

22. Tobert DG, Simon PM, Stroetz RW, Hubmayr RD. The determinants of respiratory rate during mechanical ventilation. Am J Respir Crit Care Med 1997;155(2):485-492.

23. Gattinoni L, Taccone P, Carlesso E, Marini JJ. Prone position in acute respiratory distress syndrome: rationale, indications, and limits. Am J Respir Crit Care Med 2013;188(11):1286-1293.

24. Guérin C, Reignier J, Richard JC, Beuret P, Gacouin A, Boulain T, et al. Prone positioning in severe acute respiratory distress syndrome. N Engl J Med 2013;368(23):2159-2168.

25. Bailey PP, Miller RR 3rd, Clemmer TP. Culture of early mobility in mechanically ventilated patients. Crit Care Med 2009;37(10 Suppl): S429-S435.

26. Reade MC, Finfer S. Sedation and delirium in the intensive care unit. N Engl J Med 2014;370(5):444-454.

27. Protti A, Votta E, Gattinoni L. Which is the most important strain in the pathogenesis of ventilator-induced lung injury: dynamic or static? Curr Opin Crit Care 2014;20(1):33-38.

28. Oeckler RA, Lee WY, Park MG, Kofler O, Rasmussen DL, Lee HB, et al. Determinants of plasma membrane wounding by deforming stress. Am J Physiol Lung Cell Mol Physiol 2010;299(6):L826-L833.

29. Horie T, Hildebrandt J. Dynamic compliance, limit cycles, and static equilibria of excised cat lung. J Appl Physiol 1971;31(3):423-430.

30. Villar J, Kacmarek RM, Pérez-Méndez L, Aguirre-Jaime A. A high positive end-expiratory pressure, low tidal volume ventilatory strategy improves outcome in persistent acute respiratory distress syndrome: a randomized, controlled trial. Crit Care Med 2006;34(5): 1311-1318.

31. Ferguson ND, Cook DJ, Guyatt GH, Mehta S, Hand L, Austin P, et al. High-frequency oscillation in early acute respiratory distress syndrome. N Engl J Med 2013;368(9):795-805. 